\title{
ON NORMS OF IDEMPOTENT MEASURES
}

\section{SADAHIRO SAEKI}

Let $G$ be a locally compact abelian group and $\Gamma$ its dual. The Fourier transform of a measure $\mu$ on $G$ is the function $\hat{\mu}$ on $\Gamma$ defined by

$$
\hat{\mu}(\gamma)=\int_{G}(-x, \gamma) d \mu(x) \quad(\gamma \in \Gamma) .
$$

If $\mu$ is idempotent, then $\hat{\mu}^{2}=\hat{\mu}$, so that $\hat{\mu}(\gamma)=1$ or 0 for all $\gamma \in \Gamma$. Define

$$
S(\mu)=\{\gamma \in \Gamma: \hat{\mu}(\gamma)=1\} .
$$

As is stated in [1], every idempotent measure $\mu$ on $G$ has norm 1 if and only if $S(\mu)$ is a coset of a subgroup of $\Gamma$, and if $\mu$ has norm greater than 1 , then $\|\mu\| \geqq 5^{1 / 2} / 2 \sim 1.118$. If $G$ is compact and if $d \mu(\chi)$ $=[1+(\chi, \gamma)] d m(\chi)$ for some $\gamma \in \Gamma$, with order greater than 2 , where $m$ is the Haar measure of $G$ normalized so that $\|m\|=1$, then

$$
\|\mu\|=\int_{G}|1+(\chi, \gamma)| d m(\chi) \geqq \frac{1+2^{1 / 2}}{2} \sim 1.207,
$$

the value $\left(1+2^{1 / 2}\right) / 2$ being attained when $\gamma$ has order 4 . The purpose of this paper is to show that this constant is best possible.

Theorem. If $\mu$ is an idempotent measure on $G$ with $\|\mu\|>1$, then $\|\mu\| \geqq\left(1+2^{1 / 2}\right) / 2$.

Proof. Since the support group of any idempotent measure is compact, it is sufficient to prove the theorem for compact $G$. Without loss of generality, we may also assume that $\hat{\mu}(0)=1$, i.e., that $S(\mu)$ contains the identity 0 in $\Gamma$. So, hereafter, we shall assume that $G$ is compact and $0 \in S(\mu)$.

Since $\|\mu\|>1, S(\mu)$ is not a coset in $\Gamma$, in particular, not a subgroup. Therefore we can find $\gamma_{0}, \gamma_{1}$ in $S(\mu)$ so that $\gamma_{1}-\gamma_{0} \notin S(\mu)$.

First assume $2 \gamma_{0} \in S(\mu)$, and put

$$
\begin{aligned}
f(\chi)= & \left(-\chi, \gamma_{0}\right)\left[1+\operatorname{Re}\left(-\chi, \gamma_{0}\right)\right]+\left(-\chi, \gamma_{1}\right)\left[1-\operatorname{Re}\left(-\chi, \gamma_{0}\right)\right] \\
= & \left(-\chi, \gamma_{0}\right)+\left(-\chi, \gamma_{1}\right)+\frac{1}{2}\left[\left(-\chi, 2 \gamma_{0}\right)+1\right] \\
& -\frac{1}{2}\left[\left(-\chi, \gamma_{1}+\gamma_{0}\right)+\left(-\chi, \gamma_{1}-\gamma_{0}\right)\right] .
\end{aligned}
$$

The first expression for $f$ shows that

Received by the editors January 4, 1967. 


$$
|f(\chi)| \leqq\left|1+\operatorname{Re}\left(-\chi, \gamma_{0}\right)\right|+\left|1-\operatorname{Re}\left(-\chi, \gamma_{0}\right)\right|=2,
$$

and the second expression for $f$ shows that

$$
\int_{G} f d \mu=3-\frac{1}{2} \hat{\mu}\left(\gamma_{1}+\gamma_{0}\right) \geqq 2.5 \text {. }
$$

Hence, $\|\mu\| \geqq \frac{1}{2}\left|\int_{G} f d \mu\right| \geqq 1.25$.

Next, assume that $2 \gamma_{0} \notin S(\mu)$. Let $\Gamma_{0}$ be the cyclic group generated by $\gamma_{0}, m_{0}$ the Haar measure of the annihilator of $\Gamma_{0}$, and $\mu_{0}$ the convolution of $\mu$ and $m_{0}$. Then $\mu_{0}$ is idempotent and

$$
\left\|\mu_{0}\right\|=\left\|\mu * m_{0}\right\| \leqq\|\mu\| \cdot\left\|m_{0}\right\|=\|\mu\| \text {. }
$$

Hence it will suffice to show $\left\|\mu_{0}\right\| \geqq\left(1+2^{1 / 2}\right) / 2$. Note that $S\left(\mu_{0}\right)$ $=\Gamma_{0} \cap S(\mu)$ and that $S\left(\mu_{0}\right)$ contains the elements 0 and $\gamma_{0}$ but does not contain $2 \gamma_{0}$.

Now at least one of the following conditions is satisfied for $S\left(\mu_{0}\right)$ :

(a) $S\left(\mu_{0}\right)$ is a union of two cosets of a subgroup $\Lambda$ of $\Gamma_{0}$.

(b) For some integer $p, S\left(\mu_{0}\right)$ contains the elements $p \gamma_{0},(p+1) \gamma_{0}$, and $(p+2) \gamma_{0}$.

(c) For some integer $q, S\left(\mu_{0}\right)$ contains $q \gamma_{0}$ but neither $(q+1) \gamma_{0}$ nor $(q-1) \gamma_{0}$

(d) None of the conditions (a), (b), and (c) holds.

Suppose $S\left(\mu_{0}\right)$ satisfies (a). Then it is obvious that $S\left(\mu_{0}\right)$ is the union of two cosets $\Lambda$ and $\left(\gamma_{0}+\Lambda\right)$ since $2 \gamma_{0} \notin S\left(\mu_{0}\right)$. Hence $\mu_{0}$ has the form

$$
d \mu_{0}(\chi)=\left[1+\left(\chi, \gamma_{0}\right)\right] d m_{1}(\chi),
$$

where $m_{1}$ denotes the Haar measure of the annihilator of $\Lambda$, and so $\left\|\mu_{0}\right\| \geqq\left(1+2^{1 / 2}\right) / 2$.

If the condition (b) is satisfied, there exists an integer $q$ such that the elements $q \gamma_{0},(q+1) \gamma_{0}$, and $(q+2) \gamma_{0}$ belong to $S\left(\mu_{0}\right)$ but either $(q-1) \gamma_{0}$ or $(q+3) \gamma_{0}$ does not belong to $S\left(\mu_{0}\right)$. If $(q-1) \gamma_{0} \notin S\left(\mu_{0}\right)$, put $f(\chi)=\left(-\chi(q+1) \gamma_{0}\right)\left[1+\operatorname{Re}\left(-\chi, \gamma_{0}\right)\right]+\left(-\chi, q \gamma_{0}\right)\left[1-\operatorname{Re}\left(-\chi, \gamma_{0}\right)\right]$. We have $\left\|\mu_{0}\right\| \geqq \frac{1}{2}\left|\int_{G} f d \mu_{0}\right|=2.5 / 2=1.25$. If $(q+3) \gamma_{0} \notin S\left(\mu_{0}\right)$, we can also define a function $f$ on $G$ such that $|f(\chi)| \leqq 2$ and $\int_{G} f d \mu_{0}=2.5$, and so $\left\|\mu_{0}\right\| \geqq 1.25$.

Suppose that condition (c) is the case. Define a function $f$ on $G$ by

$$
f(\chi)=\left[1+\operatorname{Re}\left(-x, \gamma_{0}\right)\right]+\left(-\chi, q \gamma_{0}\right)\left[1-\operatorname{Re}\left(-\chi, \gamma_{0}\right)\right] \text {. }
$$

We have again $\left\|\mu_{0}\right\| \geqq \frac{1}{2}\left|\int_{G} f d \mu_{0}\right| \geqq 2.5 / 2=1.25$.

Finally, suppose that the condition (d) holds. This implies that 
$S\left(\mu_{0}\right)$ is "nonperiodic" and that if $(p-1) \gamma_{0} \notin S\left(\mu_{0}\right)$ and $p \gamma_{0} \in S\left(\mu_{0}\right)$ then $(p+1) \gamma_{0} \in S\left(\mu_{0}\right)$ and $(p+2) \gamma_{0} \in S\left(\mu_{0}\right)$. In this case we may clearly assume (if necessary, replace $\gamma_{0}$ by $-\gamma_{0}$ and translate $S\left(\mu_{0}\right)$ by $p \gamma_{0}$ for some integer $p$ ) that there exists an integer $q \geqq 3$ such that $q \gamma_{0} \in S\left(\mu_{0}\right),(q+1) \gamma_{0} \in S\left(\mu_{0}\right)$ but $-p \gamma_{0} \notin S\left(\mu_{0}\right)$ whenever $1 \leqq p<q$. Put $f(\chi)=\left[1+\operatorname{Re}\left(-\chi,(q+1) \gamma_{0}\right)\right]+\left(-\chi, \gamma_{0}\right)\left[1-\operatorname{Re}\left(-\chi,(q+1) \gamma_{0}\right)\right]$.

Since $(q+2) \gamma_{0} \notin S\left(\mu_{0}\right)$ by our assumption, we have

$$
\int_{G} f d \mu_{0}=2.5+\frac{1}{2}\left[\hat{\mu}_{0}\left(-(q+1) \gamma_{0}\right)-\hat{\mu}_{0}\left(-q \gamma_{0}\right)\right] .
$$

Thus the right side in the above equality is greater than or equal to 2.5 if $\hat{\mu}_{0}\left(-(q+1) \gamma_{0}\right)-\hat{\mu}_{0}\left(-q \gamma_{0}\right) \geqq 0$. But $-q \gamma_{0} \in S\left(\mu_{0}\right)$ implies $-(q+1) \gamma_{0} \in S\left(\mu_{0}\right)$ since $-(q-1) \gamma_{0} \notin S\left(\mu_{0}\right)$. Therefore $\hat{\mu}_{0}\left(-(q+1) \gamma_{0}\right)$ $-\hat{\mu}_{0}\left(-q \gamma_{0}\right) \geqq 0$ in any case, and we have $\int_{G} f d \mu_{0} \geqq 2.5$, and $\left\|\mu_{0}\right\| \geqq 1.25$. The proof of the theorem is now completed.

\section{REFERENCE}

1. W. Rudin, Fourier analysis on groups, Interscience, New York, 1962.

Tokyo Metropolitan University 